13 Butcher KS, Parsons M, MacGregor L, Barber PA, Chalk J, Bladin C, et al: Refining the perfusion-diffusion mismatch hypothesis. Stroke 2005; 36:1153-1159.

14 Furlan AJ, Eyding D, Albers GW, Al-Rawi Y, Lees KR, Rowley HA, et al: Dose Escalation of Desmoteplase for Acute Ischemic Stroke (DEDAS): evidence of safety and efficacy 3 to 9 hours after stroke onset. Stroke 2006;37:1227-1231.

15 Wardlaw JM, Seymour J, Cairns J, Keir S, Lewis S, Sandercock P: Immediate computed tomography scanning of acute stroke is cost-effective and improves quality of life. Stroke 2004;35:2477-2483.

16 Chalela JA, Kidwell CS, Nentwich LM, Luby M, Butman JA, Demchuk $\mathrm{AM}$, et al: Magnetic resonance imaging and computed tomography in emergency assessment of patients with suspected acute stroke: a prospective comparison. Lancet 2007;369:293-298.

17 Goldstein LB, Hey LA, Laney R: North Carolina Stroke Prevention and Treatment Facilities Survey: statewide availability of programs and services. Stroke 2000;31:66-70.

18 Buckley BT, Wainwright A, Meagher T, Briley D: Audit of a policy of magnetic resonance imaging with diffusion-weighted imaging as firstline neuroimaging for in-patients with clinically suspected acute stroke. Clin Radiol 2003;58:234-237.

19 Leys D, Ringelstein B, Kaste M, Hacke W: Facilities in European hospitals treating stroke patients. Stroke 2007;38:2895-2991.

Dr. William Whiteley

Department of Clinical Neurosciences, University of Edinburgh

Western General Hospital

Edinburgh EH4 2XU (UK)

Tel. +4478 1111 3610, Fax +441313325 150

E-Mailwilliam.whiteley@ed.ac.uk

Cerebrovasc Dis 2008;25:377-380

DOI: $10.1159 / 000120689$

\section{Reversible Posterior Encephalopathy Syndrome Followed by MR Angiography-Documented Cerebral Vasospasm in Preeclampsia-Eclampsia: Report of 2 Cases}

Kiyomi Tsukimori ${ }^{\text {a }}$, Hirofumi Ochi ${ }^{\text {b }}$, Yasuo Yumoto ${ }^{a}$, Satomi Iwasaki a , Satoshi Hojo a , Tomoyuki Noguchi ${ }^{\mathrm{C}}$, Norio Wake a

Departments of a Gynecology and Obstetrics and ${ }^{b}$ Neurology, Neurological Institute and 'Department of Clinical Radiology, Graduate School of Medical Sciences, Kyushu University,

Fukuoka, Japan

\section{Introduction}

Posterior reversible encephalopathy syndrome (PRES) is a rare complication of preeclampsia-eclampsia [1]. The characteristic neuroradiological findings are multifocal and often symmetric, increased T2 or diffusion-weighted signal intensity lesions in the posterior dominant white and gray matter [2]. Diffusion tensor imaging (DWI) changes support the hypothesis that vasogenic edema is the pathophysiological mechanism underlying this syndrome [2]. Several reports, however, associate PRES with cyto- toxic edema, ischemic stroke, and angiographic vasospasm [3-6]. These findings suggest an interrelationship between PRES and cerebral vasospasm. We report two preeclamptic-eclamptic patients with clinical and neuroradiological features of PRES. In these patients, MR angiography (MRA) showed reversible vasospasm of large- and medium-sized cerebral arteries. The vasospasm was not present initially, and was preceded by the development of vasogenic edema.

\section{Case Reports}

Patient 1

A 28-year-old Japanese woman, gravida 2, para 0, with a twin pregnancy, suddenly developed a severe headache and vomiting at 35 weeks and 5 days of gestation. She had no evidence of cardiac, pulmonary, renal or cerebrovascular disease. Her blood pressure was $178 / 80 \mathrm{~mm} \mathrm{Hg}$, with proteinuria of $7.8 \mathrm{~g} / 24 \mathrm{~h}$. Neurological examination was normal. Hematological, renal and liver function tests were also normal. A diagnosis of preeclampsia was made. She was started on intravenous magnesium sulfate for seizure prophylaxis, and an emergent cesarean delivery was performed for breech presentation. Two male infants weighting 2,302 and 1,948 g were delivered, and both did well.

Four hours postoperatively, the patient grew disoriented and became obtunded. Her blood pressure was 170/110 mm Hg. A complete blood count showed no major abnormalities with a platelet count of $160,000 / \mu l$. Her electrolytes and renal function tests were normal. Brain magnetic resonance imaging (MRI) showed multifocal areas of edema with hyperintense signals on T2-weighted and fluid-attenuated inversion recovery (FLAIR) images in the bilateral frontal, parietal, and occipital gray and white matters, basal ganglia, brainstem and bilateral cerebellum (fig. 1a). These areas showed predominantly high signals on DWI that corresponded to high signal intensities on an ADC map. These findings represented vasogenic edema. However, some of the diffusion-hyperintense lesions in the bilateral basal ganglia and left cerebellum showed low-signal intensities on the ADC map. This was consistent with cytotoxic edema. An MRA at this time did not show any significant abnormalities except for an aneurysm at the right internal carotid-ophthalmic artery (fig. 1b). The patient was treated with sodium nitroprusside, nicardipine, magnesium sulfate, glycerol, and the free radical scavenger edarabone. The next day, she recovered consciousness without neurological sequelae. On postpartum day 4 , her headache had resolved and her blood pressure stabilized at $120 / 70 \mathrm{~mm} \mathrm{Hg}$.

A follow-up MRI on postpartum day 4 showed resolution of the high signal areas on FLAIR and T2-weighted images (fig. 1c); however, MRA revealed multifocal irregular narrowing of the bilateral anterior cerebral arteries (ACAs), middle cerebral arteries (MCAs), and posterior cerebral arteries (PCAs). This was consistent with vasoconstriction (fig. 1d). An extensive workup for cerebral vasculitis was negative. Additionally, microbiological, autoimmune and thrombophilia studies showed no abnormalities. On postpartum day 13, the patient's blood pressure had returned to normal without any medications, and she had no proteinuria. All neuroradiological abnormalities had resolved on a repeat brain MRI and MRA preformed on postpartum day 13. The patient had recovered completely with no neurological deficits on postpartum day 22 , and was discharged. 

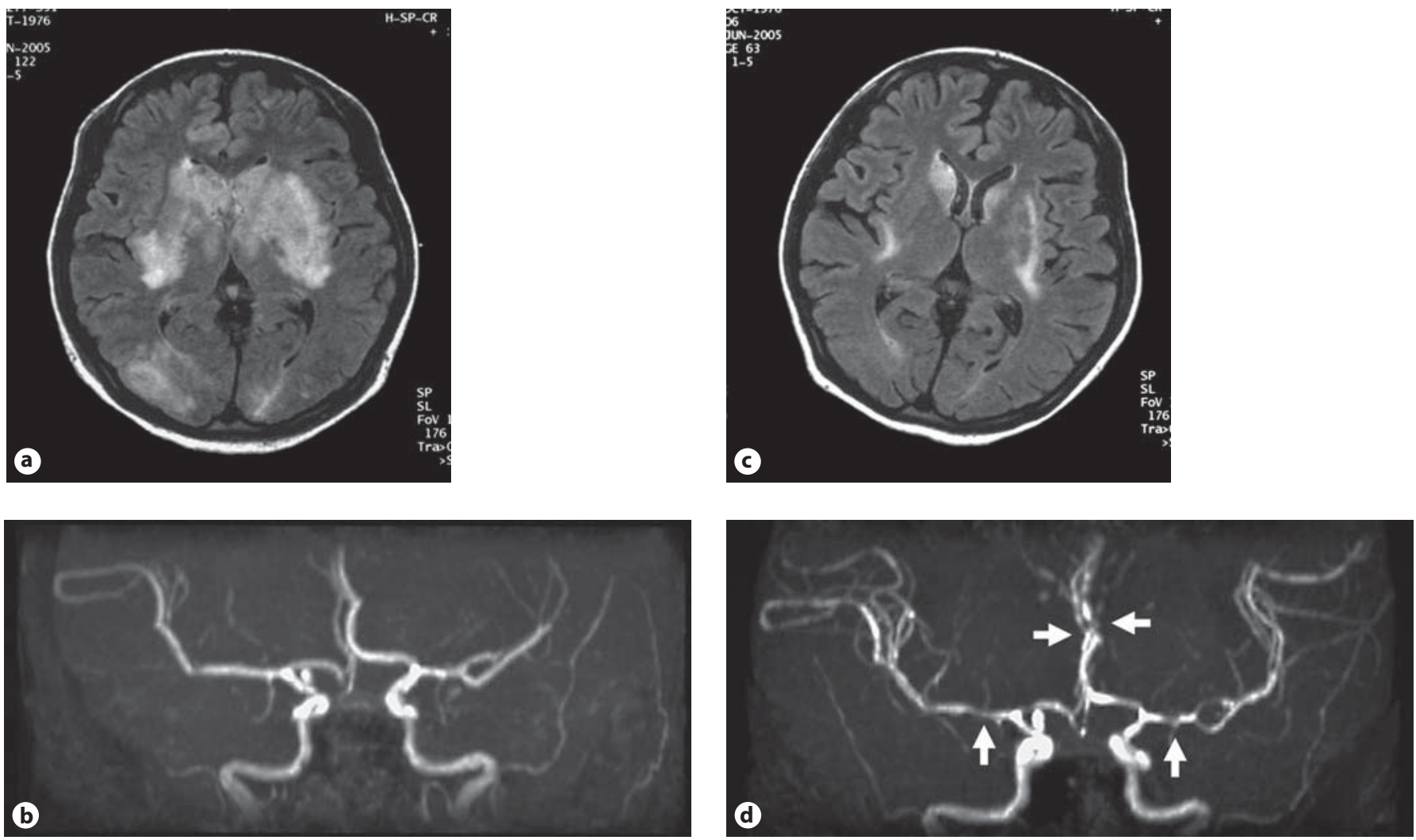

Fig. 1. Brain MRI and MRA in patient 1 on postpartum day 0 (a, b) and day 4 (c, d). a Diffusion-weighted image showing high signal intensity areas in the bilateral frontal, parietal and occipital gray and white matters, and basal ganglia. b MRA showing no significant segmental narrowing of the arteries. c Follow-up MRI in which most of the lesions have disappeared on diffusion-weighted images, except for small foci of high signal intensity in bilateral basal ganglia. d Follow-up MRA showing multifocal irregular narrowing of the bilateral ACAs and MCAs (arrows).

\section{Patient 2}

The patient, a 22-year-old Japanese woman, gravida 1, para 0 , was admitted to our hospital for spontaneous rupture of membranes at 36 weeks and 5 days of gestation. She had no evidence of cardiac, pulmonary, renal or cerebrovascular disease. Her blood pressure was $138 / 90 \mathrm{~mm} \mathrm{Hg}$ with proteinuria of $3+(1,000$ $\mathrm{mg} / \mathrm{dl}$ ). A diagnosis of preeclampsia was made. At 37 weeks and 0 days of gestation, she developed generalized tonic-clonic seizures and became obtunded during labor. Her blood pressure was 142/100 mm Hg. Neurological examination revealed a conjugate deviation of the eyes towards the right side, and mild hyperreflexia of the left extremities with pathological reflexes. Hematological, renal and liver function tests were normal. Brain MRI showed multifocal areas of hyperintense signals on FLAIR and T2-weighted images in the bilateral frontal and parietooccipital gray and white matters, and right basal ganglia (fig. 2a). These areas were hyperintense on DWI and normal to mildly increased on the ADC map. These findings were consistent with vasogenic edema. Brain MRA at this time was normal (fig. 2b), and MR ve- nography revealed no evidence of venous thrombosis. A diagnosis of eclampsia was made and an emergent cesarean delivery was performed. A male infant weighting 2,748 g was delivered and did well. Postoperatively, the patient was treated with magnesium sulfate and glycerol. The next day, she recovered consciousness without neurological sequelae. On postpartum day 6 , her blood pressure stabilized at 120/70 mm Hg.

Follow-up MRI on postpartum day 6 showed resolution of the high signal areas on FLAIR and T2-weighted images (fig. 2c). MRA, however, revealed multifocal irregular narrowing of the bilateral ACAs and PCAs (fig. 2d). The results of an extensive workup for cerebral vasculitis were negative. Microbiological, autoimmune, and thrombophilia studies likewise showed no abnormalities. She fully recovered without neurological sequelae, and was discharged on postpartum day 10. On postpartum day 40, repeat brain MRI and MRA showed complete resolution of the previous abnormalities. 

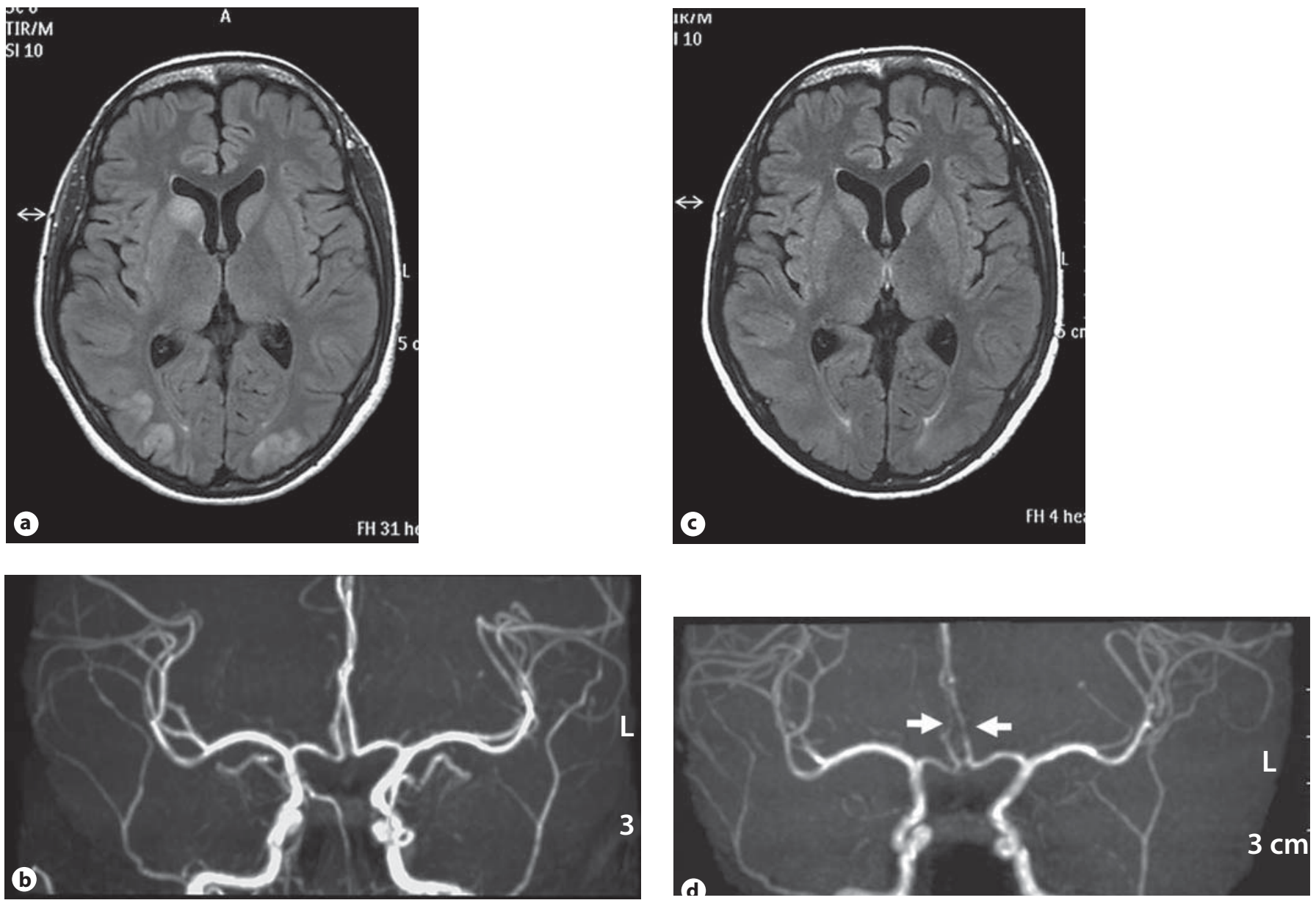

Fig. 2. Brain MRI and MRA in patient 2 at 37 weeks and 0 day of gestation (a, b) and on postpartum day 6 (c, d). a Diffusion-weighted image showing high signal intensities in the bilateral parietooccipital gray and white matters and right basal ganglia. b MRA shows no abnormality. c Follow-up MRI in which most of the high signal intensity lesions have disappeared on diffusion-weighted images. d Follow-up MRA showing multifocal irregular narrowing of the bilateral ACAs (arrows).

\section{Discussion}

Both patients presented here met the diagnostic criteria for preeclampsia in that they had both hypertension and proteinuria during pregnancy. Patient 1 developed a headache followed by loss of consciousness in the postpartum period. Patient 2 developed eclampsia or seizures in the setting of preeclampsia. The MRI findings of these patients, notably the hyperintense FLAIR and T2-weighted images, together with hyperintense DWI and normal to increased ADC, are consistent with vasogenic edema. The neurological abnormalities improved soon after treatment, and the abnormal neuroradiological findings also resolved completely within days to weeks. These clinical and MRI findings are characteristic of PRES. Small foci of cytotoxic edema showing reduced $\mathrm{ADC}$ were also detected in patient 1 . Although the edema in PRES is primarily vasogenic, concomitant cytotoxic edema has also been reported. One proposed mechanism is that endothelial damage releases potent vasoconstrictors such as endothelin, angiotensin II, and thromboxane. These mediators cause vasospasm, which results in cytotoxic edema [7]. Though the initial MRA in patient 1 did not demonstrate cerebral vasospasm, MRA may not have been sensitive enough to detect spasm in the third or higher-order arterial branches. Another possibility is that the marked vasogenic edema elevated tissue pressure. This may eventually have impaired the microcirculation, resulting in ischemic injury with cytotoxic edema [4].

The most striking neuroradiological feature in both patients is the time lag between the development of vasogenic edema and the appearance of cerebral vasospasm 4-6 days later. By this point, the edema had resolved on MRI, blood pressure had stabilized in the normal range, and all neurological signs and symptoms had resolved. PRES is characterized by reversible posteriordominant white and gray matter lesions associated with brain va- 
sogenic edema in patients with conditions including hypertensive encephalopathy, preeclampsia-eclampsia, neurotoxicity of immunosuppressive drugs, and uremic encephalopathy [1]. The pathophysiology of PRES is, however, unclear. Endothelial dysfunction and failure of cerebral autoregulation in distal arterioles and capillaries are proposed mechanisms [2]. Although most patients with PRES have normal cerebral angiograms, conventional angiograms or MRA may demonstrate cerebral vasospasm in patients with hypertensive encephalopathy associated with pregnancy or intrathecal chemotherapy $[8,9]$. Transcranial Doppler sonography has demonstrated that after the disappearance of signs and symptoms in a case of eclampsia, the flow velocity began to increase, likely due to delayed vasospasm [10]. Singhal [3] reported 4 postpartum patients with reversible cerebral vasospasm who did not meet the criteria for preeclampsia-eclampsia, and were diagnosed as having postpartum angiopathy (PPA). It is well known that cerebral vasospasm has an important role in ischemic injury, and PPA is frequently complicated by ischemic stroke. It should be noted, however, that the PPA patients reported by Singhal showed transient nonischemic brain lesions consistent with PRES. Taken together, cerebral vasospasm may occur in patients with PRES, and there is a possible interrelationship between PRES and vasospasm.

An excessive maternal systemic inflammatory response involving leukocyte activation and cytokine production has been implicated in the pathogenesis of preeclampsia-eclampsia [11]. Vasogenic edema, if significant, causes hypoxia and ischemic injury by elevating tissue pressure and impairing microcirculation [4]. Both ischemia and hypoxia also induce an inflammatory response. This response activates both leukocytes and platelets, with a subsequent release of inflammatory cytokines such as interleukin (IL)-1 $\beta$, IL- 6 and tumor necrosis factor- $\alpha$. All these cause cerebral vasospasm [12]. Thus, the hyperinflammatory state in preeclampsia-eclampsia may cause cerebral vasospasm that is followed by vasogenic edema in preeclampsia-eclampsia. Alternatively, the potent vasoconstrictors released by endothelial cell damage could mediate cerebral vasospasm [7]. Further studies are necessary to fully understand the pathophysiological features, causes, and interrelationships of cerebral vasospasm and PRES in preeclampsia-eclampsia.

\section{Acknowledgments}

This study was supported in part by a Grant-in-Aid for Scientific Research from the Japanese Society for the Promotion of Science (18591804).

\section{References}

$\checkmark 1$ Hinchey J, Chaves C, Appignani B, et al: A reversible posterior leukoencephalopathy syndrome. N Engl J Med 1996;334:494-500.

-2 Mukherjee P, McKinstry RC: Reversible posterior leukoencephalopathy syndrome: evaluation with diffusion-tensor MR imaging. Radiology 2001;219:756-765.

- 3 Singhal AB: Postpartum angiopathy with reversible posterior leukoencephalopathy. Arch Neurol 2004;61:411-416.

4 Koch S, Rabinstein A, Falcone S, et al: Diffusion-weighted imaging shows cytotoxic and vasogenic edema in eclampsia. AJNR Am J Neuroradiol 2001;22:1068-1070.

5 Dodick DW, Eross EJ, Drazkowski JF, et al: Thunderclap headache associated with reversible vasospasm and posterior leukoencephalopathy syndrome. Cephalalgia 2003;23:994-997.
6 Oehm E, Hetzel A, Els T, et al: Cerebral hemodynamics and autoregulation in reversible posterior leukoencephalopathy syndrome caused by pre-/eclampsia. Cerebrovasc Dis 2006;22:204-208.

-7 Kinoshita T, Moritani T, Shrier DA, et al: Diffusion-weighted MR imaging of posterior reversible leukoencephalopathy syndrome: a pictorial essay. Clin Imaging 2003;27:307-315.

-8 Ito T, Sakai T, Inagawa S, et al: MR angiography of cerebral vasospasm in preeclampsia. AJNR Am J Neuroradiol 1995;16:1344-1346.

-9 Henderson RD, Rajah T, Nicol AJ, et al: Posterior leukoencephalopathy following intrathecal chemotherapy with MRA-documented vasospasm. Neurology 2003;60:326-328.

10 Hashimoto H, Kuriyama Y, Naritomi H, et al: Serial assessments of middle cerebral artery flow velocity with transcranial Doppler sonography in the recovery stage of eclampsia. A case report. Angiology 1997; 48:355-358.

11 Redman CWG, Sargent IL: Pre-eclampsia, the placenta and the maternal systemic inflammatory responses - a review. Placenta 2003;24:S21S27.

12 Fassbender K, Hodapp B, Rossol S, et al: Inflammatory cytokines in subarachnoid haemorrhage: association with abnormal blood flow velocities in basal cerebral arteries. J Neurol Neurosurg Psychiatry 2001; 70:534-537.

Kiyomi Tsukimori, MD, $\mathrm{PhD}$

Department of Obstetrics and Gynecology

Graduate School of Medical Sciences, Kyushu University

Maidashi 3-1-1, Higashi-ku

Fukuoka 812-8582 (Japan)

Tel. +8192642 5394, Fax +81926425414

E-Mail tsuki@med.kyushu-u.ac.jp

Cerebrovasc Dis 2008;25:380-381

DOI: $10.1159 / 000120690$

\section{A Case of Presumed Granulomatous Carotid Stenosis}

\author{
P. Dassan ${ }^{\text {a }}$, M.M. Brown ${ }^{\text {a }}$, C. Bishop ${ }^{\text {b }}$, I. Hopkins ${ }^{c}$, D.J. Werring \\ aStroke Research Group, UCL Institute of Neurology, \\ National Hospital of Neurology and Neurosurgery, and \\ Departments of ${ }^{b}$ Vascular Surgery and ${ }^{\mathrm{C} H i s t o p a t h o l o g y, ~}$ \\ University College Hospital, London, UK
}

A 75-year-old right-handed Caucasian gentleman presented with sudden left arm weakness and dysarthria on rising from bed in the middle of the night. These symptoms resolved over 1 month. Twelve months later he was admitted to hospital for an open cholecystectomy. Two days post-operatively, he had a further attack of sudden left arm weakness lasting 1 month. Thereafter his family reported that he had memory problems and difficulty dressing himself, which subsequently improved. When seen 11 months later on our unit, neurological examination was normal. Vascular examination revealed a right carotid bruit. Fasting glucose, cholesterol, ESR, autoimmune screen, treponemal serology and chest $\mathrm{X}$-ray were normal or negative. CT of the brain showed an area of 